International Game Theory Review, Vol. 2, Nos. 2 \& 3 (2000) 155-172

(C) World Scientific Publishing Company

\title{
APPROXIMATING NASH EQUILIBRIA IN NONZERO-SUM GAMES
}

\author{
EITAN ALTMAN and ODILE POURTALLIER \\ INRIA, BP93, 06902 Sophia Antipolis, France \\ ALAIN HAURIE and FRANCESCO MORESINO* \\ Logilab-HEC, University of Geneva, \\ 40 Blvd. du Pont-d'Arve CH-1211 Geneva 4, Switzerland \\ * Cambridge University, UK
}

\begin{abstract}
This paper deals with the approximation of Nash equilibria in $m$-player games. We present conditions under which an approximating sequence of games admits nearequilibria that approximate near-equilibria in the limit game. We apply the results to two classes of games: (i) a duopoly game approximated by a sequence of matrix games, and (ii) a stochastic game played under the $S$-adapted information structure approximated by games played over a sampled event tree. Numerical illustrations show the usefulness of this approximation theory.
\end{abstract}

\section{Introduction}

Game theory has known a considerable development in the past few decades, however relatively few results have been proposed for the approximation of equilibrium solutions in nonzero-sum games. The aim of this paper is to provide conditions under which the exact or $\epsilon$-equilibrium solutions in a normal form nonzero-sum game with strategies selected in a normed space can be approximated by the exact or $\epsilon$-equilibrium solutions of a "converging" sequence of games. This question occurs very naturally in the implementation of numerical techniques for the computation of Nash equilibria or in the simplification of "large scale" games like, for example, those that are defined on a stochastic event tree when the players use the so-called $S$-adapted information structure, introduced in Haurie et al. (1987) and (1990), and further studied in Haurie and Moresino (to appear). Our approach to a theory of approximation for nonzero-sum games can be linked with the work of Whitt (1980), and references in Tidball and Altman (1996) and Tidball et al. (1997), dealing with zero-sum games, that appeared more recently.

In relatively loose terms, the general problem of approximating equilibria in nonzero-sum games can be formulated as follows. Let $G$ be an $m$-player game in normal form, with strategy sets in normed spaces. Let $G^{n}, n \in \mathbb{N}$ be a sequence of "approximating" $m$-player games with strategy sets that may be different from those used for the game $G$. We look for conditions under which: 
(i) If there exists a sequence of $\epsilon^{n}$-equilibria to the games $G^{n}, n \in \mathbb{N}, \epsilon^{n} \rightarrow \epsilon$, that corresponds, in some appropriate way to be defined shortly, to a converging sequence in the normed space of strategies for $G$, then the limit is an $\epsilon$-equilibrium in $G$. Furthermore, the sequence of $\epsilon^{n}$-equilibrium values $\bar{J}^{n}$ converges within $\epsilon$ to an equilibrium value $\bar{J}$ in the limit game [Result (1) of Theorem 3.1];

(ii) For any converging sequence $\epsilon^{n} \rightarrow \epsilon$, any $\epsilon^{\prime}>\epsilon$, for $n$ large enough, an $\epsilon^{n}$ equilibrium of $G^{n}$ corresponds to an $\epsilon^{\prime}$-equilibrium of $G$ [Result (2) of Theorem 3.1];

(iii) For any $\epsilon^{\prime}>0$, there exists an $\epsilon$-equilibrium to the game $G$ that also corresponds to an $\epsilon^{\prime}$-equilibrium to the games $G^{n}$, for $n$ large enough [Result (3) of Theorem 3.1];

(iv) For any equilibrium value $\overline{\mathbf{J}}$ of the game $G$ and for any $\epsilon>0$, there exists a converging sequence $\overline{\mathbf{J}}^{n} \rightarrow \overline{\mathbf{J}}$ such that $\overline{\mathbf{J}}^{n}$ is an $\epsilon$-equilibrium value for the game $G^{n}$ [Result (4) of Theorem 3.1].

A fundamental ingredient of a theory of approximation will be the definition of a set of correspondences that permit one to associate with a strategy vector in $G^{n}$, $n \in \mathbb{N}$, a strategy vector in $G$ and vice versa. These correspondences will have to satisfy enough regularity conditions for the convergence results to hold. This paper provides such a set of conditions.

Similar problems were studied by Cavazzuti and Pacchiarotti in (1986), and by Morgan and Raucci (1997), also dealing with the approximation of nonzerosum games. These authors use the notions of " $\epsilon$ and strict $\epsilon$-approximate Nash equilibrium" that are a further relaxation of the Nash conditions. In Cavazzuti and Pacchiarotti (1986), it is shown that the limit of a converging sequence of $\epsilon$-approximate Nash equilibria in a sequence of approximating games $G^{n}$ is an $\epsilon$ approximate Nash equilibrium in the limit game $G$. The paper by Morgan and Raucci (1997) relaxes the assumptions under which the previous result holds and shows that under appropriate assumptions, any $\epsilon$-approximate Nash equilibrium in the game $G$ can be approached by a sequence of $\epsilon$-approximate Nash equilibria in the games $G^{n}$. In these papers, all the approximating games have the same strategy sets, only the payoff functions differ and the method of proof uses convergence properties for the strategies. In the present paper, we avoid assumptions related to convergence in the strategy space. Furthermore, in Morgan and Raucci (1997), convexity properties play an important role whereas in this paper we do not use any to prove the results. However, in the present paper, the regularity properties and the uniform convergence assumptions on the payoff functions are quite restrictive, so we cannot claim to be more general than Cavazzuti and Pacchiarotti (1986), and Morgan and Raucci (1997). Our set of conditions are different and may prove to be easier to verify for some dynamic games.

The paper is organised as follows. In Sec. 2, we present as a motivating example the approximation of a simple duopoly game via a sequence of bimatrix games. 
This permits us to illustrate the use of correspondences between the limit and the approximating games, and to observe the convergence of Nash equilibria solutions. In Sec. 3, we derive the main convergence theorems. In Sec. 4, we apply the theory to the approximation of a static Nash equilibrium in a concave continuous game by Nash equilibria in $m$-matrix games. In Sec. 5 , we apply the theory to a class of stochastic games with the $S$-adapted information structure.

\section{A Motivating Example}

Consider a duopoly game where two firms supply a market characterised by the (inverse) demand law

$$
p\left(q_{1}, q_{2}\right)=\frac{\alpha}{q_{1}+q_{2}+\beta}-\gamma
$$

with $\alpha, \beta>0, \gamma \geq 0$, where $q_{i} \in\left[0, q_{i}^{\max }\right]$ is the quantity supplied by firm $i$ and $p\left(q_{1}, q_{2}\right)$ is the market clearing price. The firms payoff functions are given by

$$
J_{i}\left(q_{1}, q_{2}\right)=\left(p\left(q_{1}, q_{2}\right)-\kappa_{i} q_{i}\right) q_{i}, \quad i=1,2,
$$

where $\kappa_{i}$ is a positive parameter, representing the unit production cost of firm $i$.

We have solved different problems with parameters summarised in Table 1. For all these problems, the existence and uniqueness conditions of Rosen (1965) are satisfied. We compare the equilibrium pairs in the continuous duopoly game with the equilibrium pairs for the approximating games obtained when one discretises the interval $[0,10]$ with a grid mesh 0.1. Associated with each discretisation is defined a bimatrix game, the equilibria of which are computed via the algorithm of Audet et al. (1999) (this algorithm computes all the equilibria in a bimatrix game). The following table shows the results obtained. The equilibrium strategies for the duopoly games are given with a precision of $10^{-4}$. We notice that all the approximating games, except for $E 3$ have a single equilibrium that is very close to the duopoly solution. In $E 3$, the approximating game has three equilibria, the third one involving mixed strategies. If one takes the grid's mesh equal to 0.05 , the approximating game has one equilibrium with both controls equal to 0.905 . This clearly illustrates a convergence property of the sequence of approximating matrix

Table 1. The six games considered.

\begin{tabular}{cccccc}
\hline \hline Games & $\alpha$ & $\beta$ & $\gamma$ & $\kappa_{1}$ & $\kappa_{2}$ \\
\hline E1 & 20 & 1 & 0 & 1.0 & 1.0 \\
E2 & 20 & 1 & 0 & 1.0 & 1.1 \\
E3 & 10 & 2 & 1 & 1.0 & 1.0 \\
E4 & 10 & 2 & 1 & 1.0 & 1.1 \\
E5 & 40 & 5 & 2 & 1.0 & 1.0 \\
E6 & 40 & 5 & 2 & 1.0 & 1.1 \\
\hline \hline
\end{tabular}


Table 2. The equilibria of the six games considered.

\begin{tabular}{|c|c|c|c|c|c|c|c|c|c|c|}
\hline \multirow[t]{2}{*}{ Example } & \multicolumn{2}{|c|}{ Limit game } & \multicolumn{8}{|c|}{ Approximating game } \\
\hline & $q_{1}^{*}$ & $q_{2}^{*}$ & \multicolumn{4}{|c|}{$q_{1}^{*}$} & \multicolumn{4}{|c|}{$q_{2}^{*}$} \\
\hline E1 & 4.9580 & 4.9580 & \multicolumn{4}{|c|}{4.96} & \multicolumn{4}{|c|}{4.96} \\
\hline \multirow[t]{2}{*}{$\mathrm{E} 2$} & 4.9905 & 4.4459 & \multicolumn{4}{|c|}{4.99} & \multicolumn{4}{|c|}{4.45} \\
\hline & & & \multicolumn{4}{|c|}{0.90} & \multicolumn{4}{|c|}{0.91} \\
\hline \multirow[t]{2}{*}{ E3 } & 0.9057 & 0.9057 & \multicolumn{4}{|c|}{0.91} & \multicolumn{4}{|c|}{0.90} \\
\hline & & & 0.90 & $16.7 \%$ & 0.91 & $83.3 \%$ & 0.90 & $16.7 \%$ & 0.91 & $83.3 \%$ \\
\hline $\mathrm{E} 4$ & 0.9413 & 0.8012 & \multicolumn{4}{|c|}{0.94} & \multicolumn{4}{|c|}{0.80} \\
\hline E5 & 2.5000 & 2.5000 & \multicolumn{4}{|c|}{2.50} & \multicolumn{4}{|c|}{2.50} \\
\hline E6 & 2.5604 & 2.3165 & \multicolumn{4}{|c|}{2.56} & \multicolumn{4}{|c|}{2.32} \\
\hline
\end{tabular}

games. It is important to remark that the convergence illustrated is not what we really need in practice, since we want to be sure that, when using a strategy pair that is an equilibrium for the approximating matrix game, one also obtains an $\epsilon$ equilibrium for the limit game. Indeed, in this particular case, the continuity of the payoff functions of the duopoly game will provide the needed result. This gives a clue on what we intend to do for a more general class of games.

\section{Convergence of Nash Equilibria}

\subsection{Definitions and notations}

Let $\mathcal{M}=\{1,2, \ldots, m\}$ be the set of players. An $m$-player nonzero-sum game is defined by the data $G=(\mathbf{J}, \mathbf{U})=\left(J_{1}, J_{2}, \ldots, J_{m}, U_{1}, U_{2}, \ldots, U_{m}\right)$, where $U_{i}$ and $J_{i}$ : $\mathbf{U} \rightarrow \mathbb{R}, i \in \mathcal{M}$, are the $i$ th player's strategy set and payoff function, respectively. An element $\mathbf{u} \in \mathbf{U}$ is called an $m$-dimensional strategy vector. We denote $u_{i} \in U_{i}$ the $i$ th component of $\mathbf{u}$ and $\mathbf{u}_{-i} \in \mathbf{U}_{-i}, i \in \mathcal{M}$, the $(m-1)$-dimensional vector obtained by removing the $i$ th component of vector $\mathbf{u}$. We denote $\left(v_{i}, \mathbf{u}_{-i}\right)$, the $m$-dimensional strategy formed by appending the $i$ th component $v_{i}$ to the $(m-1)$ dimensional vector $\mathbf{u}_{-i}$.

Definition 3.1. A Nash equilibrium, or more simply an equilibrium, is a strategy vector $\overline{\mathbf{u}}=\left(\bar{u}_{1}, \ldots, \bar{u}_{m}\right)$ such that

$$
\bar{J}_{i}=J_{i}(\overline{\mathbf{u}})=\max _{u_{i} \in U_{i}} J_{i}\left(u_{i}, \overline{\mathbf{u}}_{-i}\right), \quad i \in \mathcal{M} .
$$

As we plan to develop a theory of approximation, we also consider $\epsilon$-equilibria.

Definition 3.2. Given $\epsilon>0$, an $\epsilon$-equilibrium is a strategy vector $\overline{\mathbf{u}}^{\epsilon}=$ $\left(\bar{u}_{1}^{\epsilon}, \ldots, \bar{u}_{m}^{\epsilon}\right)$ such that

$$
\forall i \in \mathcal{M} \forall u_{i} \in U_{i}, \quad J_{i}\left(u_{i}, \overline{\mathbf{u}}_{i}^{\epsilon}\right) \leq J_{i}\left(\overline{\mathbf{u}}^{\epsilon}\right)+\epsilon .
$$


Remark 3.1. Note that, when the context is obvious, we will often omit the $\epsilon$ in the strategy notation for an $\epsilon$-equilibrium.

We assume that each strategy set $U_{i}, i \in \mathcal{M}$, of the game $G$ is a closed subset in a normed space and the following continuity conditions hold for each payoff function.

Assumption 3.1. In the game $G$, for each player $i \in \mathcal{M}$, the payoff function satisfies

(A1a) $J_{i}$ is continuous in $\mathbf{u}_{-i}$, uniformly for all $v_{i}$, i.e.,

$$
\begin{gathered}
\forall \epsilon, \forall \tilde{\mathbf{u}}_{-i}=\left\{\mathbf{u}_{-i k}\right\}_{k \in \mathbb{N}} \rightarrow \mathbf{u}_{-i}, \exists K\left(\epsilon, \tilde{\mathbf{u}}_{-i}\right), \quad \text { s.t. } \forall k \geq K\left(\epsilon, \tilde{\mathbf{u}}_{-i}\right), \forall u_{i}, \\
\left|J_{i}\left(v_{i}, \mathbf{u}_{-i k}\right)-J_{i}\left(v_{i}, \mathbf{u}_{-i}\right)\right| \leq \epsilon .
\end{gathered}
$$

(A1b) $J_{i}$ is upper-semicontinuous in $u_{i}$, uniformly for all $\mathbf{v}_{-i} \in \mathbf{U}_{-i}$ i.e.,

$$
\begin{gathered}
\forall \epsilon, \forall \tilde{u}_{i}=\left\{u_{i k}\right\}_{k \in \mathbb{N}} \rightarrow u_{i}, \exists K\left(\epsilon, \tilde{u}_{i}\right), \quad \text { s.t. } \forall k \geq K\left(\epsilon, \tilde{u}_{i}\right), \forall \mathbf{v}_{-i} \in \mathbf{U}_{-i}, \\
J_{i}\left(u_{i k}, \mathbf{v}_{-i}\right) \leq J_{i}\left(u_{i}, \mathbf{v}_{-i}\right)+\epsilon .
\end{gathered}
$$

Remark 3.2. Usually, for establishing existence of equilibria one assumes continuity of the payoff functions and compactness of the strategy sets. Indeed this implies the above assumptions.

\subsection{Approximating games}

We consider a sequence of "approximating" $m$-player games

$$
G^{n}=\left(\mathbf{J}^{\mathbf{n}}, \mathbf{U}^{\mathbf{n}}\right)=\left(J_{1}^{n}, J_{2}^{n}, \ldots, J_{m}^{n}, U_{1}^{n}, U_{2}^{n}, \ldots, U_{m}^{n}\right), \quad n=1,2, \ldots, \infty,
$$

where $U_{i}^{n}$ (resp. $J_{i}^{n}$ ) is the set of strategies (resp. the payoff function) of player $i$ for the $n$th game. The strategy sets in the game $G^{n}$ can be very different from those defined for the limit game $G$. For example, $U_{i}^{n}$ is a finite set or its convex hull, whereas $U_{i}$ is a general convex set. So we introduce a class of mappings $\pi_{i}^{n}$ and $\sigma_{i}^{n}$ that will permit us to establish a correspondence between strategies in $G^{n}$ and strategies in $G$ and vice versa. In order for the games $G^{n}$ to approximate the game $G$, there must be some "continuity properties" satisfied. We suppose the following

Assumption 3.2. For each $n \in \mathbb{N}$, there exist functions $\pi^{n}=\left(\pi_{1}^{n}, \ldots, \pi_{m}^{n}\right)$ and $\sigma^{n}=\left(\sigma_{1}^{n}, \ldots, \sigma_{m}^{n}\right)$ where $\pi_{i}^{n}: U_{i}^{n} \rightarrow U_{i}$ and $\sigma_{i}^{n}: U_{i} \rightarrow U_{i}^{n}, \forall i \in \mathcal{M}$, for which the following conditions hold:

(A2) $\varliminf_{n \rightarrow+\infty}\left[J_{i}^{n}\left(\sigma_{i}^{n}\left(v_{i}\right), \mathbf{u}_{-i}^{n}\right)-J_{i}\left(v_{i}, \pi_{-i}^{n}\left(\mathbf{u}_{-i}^{n}\right)\right)\right] \geq 0$ uniformly in the sequence $\left\{\mathbf{u}_{-i}^{n}\right\} \in \mathbf{U}_{-i}^{n}$ and in $v_{i} \in U_{i}$, i.e. such that

$$
\begin{gathered}
\forall \epsilon, \exists N(\epsilon), \forall\left\{\mathbf{u}_{-i}^{n}\right\}_{n \in \mathbb{N}} \in \mathbf{U}_{-i}^{n}, \forall v_{i} \in U_{i}, \\
J_{i}^{n}\left(\sigma_{i}^{n}\left(v_{i}\right), \mathbf{u}_{-i}^{n}\right) \geq J_{i}\left(v_{i}, \pi_{-i}^{n}\left(\mathbf{u}_{-i}^{n}\right)\right)-\epsilon .
\end{gathered}
$$

(A3) $\varliminf_{n \rightarrow+\infty}\left[J_{i}^{n}\left(\sigma^{n}(\mathbf{u})\right)-J_{i}(\mathbf{u})\right] \geq 0$, for any $\mathbf{u} \in \mathbf{U}$. 
(A4) $\varlimsup_{n \rightarrow+\infty}\left[J_{i}^{n}\left(u_{i}^{n}, \sigma_{-i}^{n}\left(\mathbf{v}_{-i}\right)\right)-J_{i}\left(\pi_{i}^{n}\left(u_{i}^{n}\right), \mathbf{v}_{-i}\right)\right] \leq 0$, uniformly in the sequence $\left\{u_{i}^{n}\right\}_{n \in \mathbb{N}} \in U_{i}^{n}$ and in $\mathbf{v}_{-i} \in \mathbf{U}_{-i}$, i.e. such that

$$
\begin{gathered}
\forall \epsilon, \exists N(\epsilon), \forall \mathbf{v}_{-i} \in \mathbf{U}_{-i}, \forall\left\{u_{i}^{n}\right\}_{n \in \mathbb{N}} \in U_{i}^{n}, \\
J_{i}^{n}\left(u_{i}^{n}, \sigma_{-i}^{n}\left(\mathbf{v}_{-i}\right)\right) \leq J_{i}\left(\pi_{i}^{n}\left(u_{i}^{n}\right), \mathbf{v}_{-i}\right)+\epsilon .
\end{gathered}
$$

(A5) $\varlimsup_{n \rightarrow+\infty}\left[J_{i}^{n}\left(\mathbf{u}^{n}\right)-J_{i}\left(\pi^{n}\left(\mathbf{u}^{n}\right)\right)\right] \leq 0$ for any sequence $\mathbf{u}^{n} \in \mathbf{U}^{n}$.

(A6) One of the following conditions hold

$$
\begin{aligned}
& \text { a : } \varliminf_{n \rightarrow+\infty}\left[J_{i}^{n}\left(\sigma^{n}(\mathbf{u})\right)-J_{i}(\mathbf{u})\right] \leq 0 \quad \text { for any } \mathbf{u} \in \mathbf{U} . \\
& \text { b : } \varlimsup_{n \rightarrow \infty} J\left(\pi^{n}\left(\sigma^{n}(\mathbf{u})\right)\right) \leq J(\mathbf{u}) \\
& \text { c : } \lim _{n \rightarrow+\infty} \pi^{n}\left(\sigma^{n}(\mathbf{u})\right)=\mathbf{u} .
\end{aligned}
$$

Remark 3.3. In the case where for all $n$ the sets $U_{i}^{n}$ are identical to the set $U_{i}$, and where the functions $\pi_{i}^{n}$ and $\sigma_{i}^{n}$ are taken as the identity, then assumptions (A2) to (A6) are a slight relaxation to the uniform convergence of $J_{i}^{n}$ to $J_{i}$.

Definition 3.3. Consider a game $G=(\mathbf{J}, \mathbf{U})$ that satisfies Condition (A1). We say that a sequence of games $G^{n}=\left(\mathbf{J}^{n}, \mathbf{U}^{n}\right)$ is a good approximating sequence for the game $G$, if there exist functions $\pi^{n}$ and $\sigma^{n}$ such that conditions (A2) to (A6) hold.

\subsection{Main results}

Theorem 3.1. Let $\left\{G^{n}\right\}_{n \in \mathbb{N}}$ be a good approximating sequence for the game $G$. Then the following results hold true.

Result (1). Let $\overline{\mathbf{u}}^{n}=\left(\bar{u}_{1}^{n}, \bar{u}_{2}^{n} \cdots \bar{u}_{m}^{n}\right) \in U^{n}, n=1,2, \ldots$, be a sequence of $\epsilon^{n}$-Nash equilibria for the respective games $G^{n}$. If $\pi^{n}\left(\overline{\mathbf{u}}^{n}\right)$ converges to $\overline{\mathbf{u}} \in \mathbf{U}$ and $\epsilon^{n}$ converges to $\bar{\epsilon}$, then for any $\epsilon>\bar{\epsilon}, \overline{\mathbf{u}}$ is an $\epsilon$-Nash equilibrium for the game $G$, and the equilibria values converge within $\bar{\epsilon}$, i.e.

$$
\varlimsup_{n \rightarrow \infty} J_{i}^{n}\left(\overline{\mathbf{u}}^{n}\right) \leq J_{i}(\overline{\mathbf{u}}) \leq \varliminf_{n \rightarrow \infty} J_{i}^{n}\left(\overline{\mathbf{u}}^{n}\right)+\bar{\epsilon} .
$$

Result (2). Consider a sequence of real numbers $\left\{\epsilon^{n}\right\}$ that converges to $\bar{\epsilon}$. For each $n$, let $\overline{\mathbf{u}}^{n}=\left(\bar{u}_{1}^{n}, \bar{u}_{2}^{n} \cdots \bar{u}_{m}^{n}\right)$ be an $\epsilon^{n}$-Nash equilibrium for the game $G^{n}$. Then for any $\epsilon>\bar{\epsilon}$, there exists $N$ such that, for all $n>N, \pi^{n}\left(\overline{\mathbf{u}}^{n}\right)$ is an $\epsilon$-Nash equilibrium for the limit game $G$.

Result (3). Let $\bar{\epsilon} \geq 0$ be given and let $\overline{\mathbf{u}}$ be an $\bar{\epsilon}$-Nash equilibrium for the limit game $G$. Then for any $\epsilon>\bar{\epsilon}$, there exists an integer $N$ such that for any $n>N$, $\sigma^{n}(\overline{\mathbf{u}})$ is an $\epsilon$-Nash equilibrium for the game $G^{n}$.

Result (4). Suppose that the limit game $G$ admits a Nash equilibrium (not necessarily unique). Let $\overline{\mathbf{J}}$ be the payoff vector value associated with that equilibrium. Then for any $\epsilon>0$, there exists a sequence $\overline{\mathbf{J}}^{n}$ that converges to $\overline{\mathbf{J}}$, and such that $\overline{\mathbf{J}}^{n}$ is the payoff vector value associated with an $\epsilon$-equilibrium for the game $G^{n}$. 
Remark 3.4. Result (1) is in the spirit of Cavazzuti and Pacchiarotti (1986), and Morgan and Raucci (1999). Results (2) to (4) are quite different since they do not involve the convergence in the strategy sets.

Proof. Result (1). We first prove that $\overline{\mathbf{u}}$ is an $\epsilon$-equilibrium, i.e. for all $i$ in $\mathcal{M}$, all $\epsilon>\bar{\epsilon}$,

$$
\sup _{u_{i}} J_{i}\left(u_{i}, \overline{\mathbf{u}}_{-i}\right) \leq J_{i}\left(\bar{u}_{i}, \overline{\mathbf{u}}_{-i}\right)+\epsilon=J_{i}(\overline{\mathbf{u}})+\epsilon .
$$

By the fact that the sequence $\pi_{-i}^{n}\left(\overline{\mathbf{u}}_{-i}^{n}\right)$ converges to $\overline{\mathbf{u}}_{-i}$ and the lower continuity of $J_{i}$ in $\mathbf{u}_{-i}$ (implied by Condition (A1a) in Assumption 3.1), for all $\epsilon_{2}>0$ sufficiently small, one can find $N_{i 2}$ such that for all $n>N_{i 2}$ and all $u_{i} \in U_{i}$,

$$
J_{i}\left(u_{i}, \overline{\mathbf{u}}_{-i}\right) \leq J_{i}\left(u_{i}, \pi_{-i}^{n}\left(\overline{\mathbf{u}}_{-i}^{n}\right)\right)+\epsilon_{2} .
$$

By the fact that $\overline{\mathbf{u}}^{n}$ is an $\epsilon^{n}$-equilibrium, and by Condition (A2) of Assumption 3.2, it follows that for $\epsilon_{3}>0$ arbitrarily small, one can find $N_{i 3}$ such that for all $n>N_{i 3}$,

$$
J_{i}\left(u_{i}, \pi_{-i}^{n}\left(\overline{\mathbf{u}}_{-i}^{n}\right)\right) \leq J_{i}^{n}\left(\sigma_{i}^{n}\left(u_{i}\right), \overline{\mathbf{u}}_{-i}^{n}\right)+\epsilon_{3} .
$$

As $\overline{\mathbf{u}}^{n}$ is an $\epsilon^{n}$-Nash equilibrium for the game $G^{n}$, the following inequality holds

$$
J_{i}^{n}\left(\sigma_{i}^{n}\left(u_{i}\right), \overline{\mathbf{u}}_{-i}^{n}\right) \leq J_{i}^{n}\left(\overline{\mathbf{u}}^{n}\right)+\epsilon^{n} .
$$

By (A5), for any $\epsilon_{4}>0$ arbitrarily small there exists $N_{i 4}$ such that for all $n>N_{i 4}$,

$$
J_{i}^{n}\left(\overline{\mathbf{u}}^{n}\right) \leq J_{i}\left(\pi^{n}\left(\overline{\mathbf{u}}^{n}\right)\right)+\epsilon_{4} .
$$

From Eqs. (3) to (6), the upper semi-continuity of $J_{i}$ in all its arguments, the convergence of the sequence $\pi^{n}\left(\overline{\mathbf{u}}^{n}\right)$ to $\overline{\mathbf{u}}$ and the convergence of the sequence $\epsilon^{n}$ to $\bar{\epsilon}$, it follows that, for any $n \geq \max \left(N_{i 2}, N_{i 3}, N_{i 4}, i \in \mathcal{M}\right)$, the following inequality is true

$$
\sup _{u_{i}} J_{i}\left(u_{i}, \overline{\mathbf{u}}_{-i}\right) \leq J_{i}(\overline{\mathbf{u}})+\bar{\epsilon}+\epsilon^{\prime}, \quad \forall i \in \mathcal{M} \quad \text { with } \epsilon^{\prime}=\epsilon_{2}+\epsilon_{3}+\epsilon_{4} .
$$

This shows that $\overline{\mathbf{u}}$ is an $\epsilon$-Nash equilibrium for the game $G$, with $\epsilon=\bar{\epsilon}+\epsilon^{\prime}$. Since $\epsilon_{2}, \epsilon_{3}$ and $\epsilon_{4}$ can be chosen arbitrarily small, the result follows.

To prove the convergence of the values $J^{n}\left(\overline{\mathbf{u}}^{n}\right)$ to $J(\overline{\mathbf{u}})$, consider the sequence $J_{i}\left(\bar{u}_{i}, \pi_{-i}^{n}\left(\overline{\mathbf{u}}_{-i}^{n}\right)\right)$. By Condition (A2) and since $\overline{\mathbf{u}}^{n}$ is $\epsilon^{n}$ equilibrium for the game $G^{n}$, one has for any $\varepsilon>0$ and $n$ sufficiently large

$$
J_{i}\left(\bar{u}_{i}, \pi_{-i}^{n}\left(\overline{\mathbf{u}}_{-i}^{n}\right)\right) \leq J_{i}^{n}\left(\sigma_{i}^{n}\left(\bar{u}_{i}\right), \overline{\mathbf{u}}_{-i}^{n}\right)+\varepsilon \leq J_{i}^{n}\left(\overline{\mathbf{u}}^{n}\right)+\epsilon^{n}+\varepsilon .
$$

By Condition (A1a) of Assumption 3.1, we can write at the limit

$$
J_{i}(\overline{\mathbf{u}})=\lim _{n \rightarrow \infty} J_{i}\left(\bar{u}_{i}, \pi_{-i}^{n}\left(\overline{\mathbf{u}}_{-i}^{n}\right)\right) \leq \varliminf_{n \rightarrow \infty} J_{i}^{n}\left(\overline{\mathbf{u}}^{n}\right)+\bar{\epsilon} .
$$

By (6), (A1a) and (A1b), we also have

$$
\varlimsup_{n \rightarrow \infty} J_{i}^{n}\left(\overline{\mathbf{u}}^{n}\right) \leq J_{i}(\overline{\mathbf{u}}) .
$$

Therefore, we conclude from (7) and (8) that Inequality (1) is satisfied. 
Result (2). Let $\epsilon^{\prime}=\epsilon-\bar{\epsilon}>0$. By (A2), we know that there exists $N_{i 1}$ such that for any $n>N_{i 1}$ we have for all $u_{i} \in U_{i}$,

$$
J_{i}\left(u_{i}, \pi_{-i}^{n}\left(\overline{\mathbf{u}}_{-i}^{n}\right)\right) \leq J_{i}^{n}\left(\sigma_{i}^{n}\left(u_{i}\right), \overline{\mathbf{u}}_{-i}^{n}\right)+\frac{\epsilon^{\prime}}{3} .
$$

Since $\overline{\mathbf{u}}^{n}$ is an $\epsilon^{n}$-Nash equilibrium in $G^{n}$, and since the sequence $\epsilon^{n}$ converges to $\bar{\epsilon}$, there exists $N_{i 2}$ such that for any $n>N_{i 2}$ and all $u_{i} \in U_{i}$,

$$
J_{i}^{n}\left(\sigma_{i}^{n}\left(u_{i}\right), \overline{\mathbf{u}}_{-i}^{n}\right) \leq J_{i}^{n}\left(\overline{\mathbf{u}}^{n}\right)+\frac{\epsilon^{\prime}}{3}+\bar{\epsilon} .
$$

Now by (A5), there exists $N_{i 3}$ such that for any $n>N_{i 3}$,

$$
J_{i}^{n}\left(\overline{\mathbf{u}}^{n}\right) \leq J_{i}\left(\pi^{n}\left(\overline{\mathbf{u}}^{n}\right)\right)+\frac{\epsilon^{\prime}}{3} .
$$

Considering Eqs. (9) to (11) together, it follows that for any $n>\max \left(N_{i 1}\right.$, $\left.N_{i 2}, N_{i 3}, i \in \mathcal{M}\right)$, we have for any $i \in \mathcal{M}$,

$$
\sup _{u_{i} \in U_{i}} J_{i}\left(u_{i}, \pi_{-i}^{n}\left(\overline{\mathbf{u}}_{-i}^{n}\right)\right) \leq J_{i}\left(\pi^{n}\left(\overline{\mathbf{u}}^{n}\right)\right)+\epsilon .
$$

This ends the proof of Result (2).

Result (3). Let $\epsilon^{\prime}=\epsilon-\bar{\epsilon}>0$. By (A4), there exists $N_{i 2}$ such that for any $n>N_{i 2}$ and $u_{i}^{n} \in U_{i}^{n}$,

$$
J_{i}^{n}\left(u_{i}^{n}, \sigma_{-i}^{n}\left(\overline{\mathbf{u}}_{-i}\right)\right) \leq J_{i}\left(\pi_{i}^{n}\left(u_{i}^{n}\right), \overline{\mathbf{u}}_{-i}\right)+\frac{\epsilon^{\prime}}{2} .
$$

Since $\overline{\mathbf{u}}$ is an $\bar{\epsilon}$-Nash equilibrium, the following inequality holds for all $u_{i}^{n}$

$$
J_{i}\left(\pi_{i}^{n}\left(u_{i}^{n}\right), \overline{\mathbf{u}}_{-i}\right) \leq J_{i}(\overline{\mathbf{u}})+\bar{\epsilon},
$$

and, by (A3), there exists $N_{i 3}$ such that, for any $n>N_{i 3}$,

$$
J_{i}(\overline{\mathbf{u}}) \leq J_{i}^{n}\left(\sigma^{n}(\overline{\mathbf{u}})\right)+\frac{\epsilon^{\prime}}{2} .
$$

Considering together Eqs. (13) to (15) for any $n>\max \left(N_{i 2}, N_{i 3}, i \in \mathcal{M}\right)$, we obtain that

$$
\sup _{u_{i}^{n} \in U_{i}^{n}} J_{i}^{n}\left(u_{i}^{n}, \sigma_{-i}^{n}\left(\overline{\mathbf{u}}_{-i}\right)\right) \leq J_{i}^{n}\left(\sigma^{n}(\overline{\mathbf{u}})\right)+\epsilon,
$$

and this ends the proof of Result (3).

Result (4). Let $\overline{\mathbf{u}}$ be the Nash equilibrium strategy profile of game $G$ associated with the Nash equilibrium value $\overline{\mathbf{J}}$. For any $\epsilon_{1}>0$, according to Result (3) established above, there exists $N_{1}$ such that for any $n \geq N_{1}, \sigma^{n}(\overline{\mathbf{u}})$ is an $\epsilon_{1}$-Nash equilibrium for the game $G^{n}$. Denote $\bar{J}_{i}^{n}=J_{i}^{n}\left(\sigma^{n}(\overline{\mathbf{u}})\right)$. According to Assumption (A3), for any $\epsilon_{2}>0$, we can choose $n$ sufficiently large, say $n \geq N_{2}$, so that for each $i$

$$
\bar{J}_{i}-\bar{J}_{i}^{n}=J_{i}(\overline{\mathbf{u}})-J_{i}^{n}\left(\sigma^{n}(\overline{\mathbf{u}})\right) \leq \epsilon_{2} .
$$

We also need an estimate for $\bar{J}_{i}^{n}-\bar{J}_{i}$. 
If Condition (A6a) holds, then for any $\epsilon_{2^{\prime}}$, we can choose $n$ sufficiently large, $n \geq N_{2^{\prime}}$ such that for each $i$,

$$
\bar{J}_{i}^{n}-\bar{J}_{i}=J_{i}^{n}\left(\sigma^{n}(\overline{\mathbf{u}})\right)-J_{i}(\overline{\mathbf{u}}) \leq \epsilon_{2^{\prime}},
$$

which provides the desired inequality.

If condition (A6a) does not hold, then we rely on (A6b) or (A6c). Since $\sigma^{n}(\overline{\mathbf{u}})$ is an $\epsilon_{1}$-Nash equilibrium for the game $G^{n}, n \geq N_{1}$, by Result (2) established above, we know that for any $\epsilon_{3}>\epsilon_{1}$, there exists $N_{3}$ such that, for any $n \geq N_{3}, \pi^{n}\left(\sigma^{n}(\overline{\mathbf{u}})\right)$ is an $\epsilon_{3}$-Nash equilibrium for the game $G$. Using Assumption (A5) we get that for any $\epsilon_{4}$, there exists $N_{4}$ such that for any $n>N_{4}$, for each $i$

$$
J_{i}^{n}\left(\sigma^{n}(\overline{\mathbf{u}})\right)-J_{i}\left(\pi^{n}\left(\sigma^{n}(\overline{\mathbf{u}})\right)\right) \leq \epsilon_{4} .
$$

If Condition (A6b) is satisfied, then for each player $i$ and any $\epsilon_{5}>0$, there exists $N_{5}$ such that for any $n \geq N_{5}$,

$$
\bar{J}_{i}^{n}-\bar{J}_{i}=J_{i}^{n}\left(\sigma^{n}(\overline{\mathbf{u}})\right)-J_{i}\left(\pi^{n}\left(\sigma^{n}(\overline{\mathbf{u}})\right)\right)+J_{i}\left(\pi^{n}\left(\sigma^{n}(\overline{\mathbf{u}})\right)\right)-J_{i}(\overline{\mathbf{u}}) \leq \epsilon_{4}+\epsilon_{5},
$$

which provides again the desired inequality, since we can choose $\epsilon=\max \left\{\epsilon_{1}, \epsilon_{2}, \epsilon_{4}+\right.$ $\left.\epsilon_{5}\right\}$ arbitrarily small.

If Condition (A6c) holds, together with (A1a) and (A1b) it implies that there exists $N_{5}$, such that for any $n \geq N_{5}$,

$$
\begin{aligned}
\bar{J}_{i}^{n}-\bar{J}_{i}= & J_{i}^{n}\left(\sigma^{n}(\overline{\mathbf{u}})\right)-J_{i}\left(\pi^{n}\left(\sigma^{n}(\overline{\mathbf{u}})\right)\right)+J_{i}\left(\pi^{n}\left(\sigma^{n}(\overline{\mathbf{u}})\right)\right)-J_{i}(\overline{\mathbf{u}}) \\
= & J_{i}^{n}\left(\sigma^{n}(\overline{\mathbf{u}})\right)-J_{i}\left(\pi^{n}\left(\sigma^{n}(\overline{\mathbf{u}})\right)\right)+J_{i}\left(\pi^{n}\left(\sigma^{n}(\overline{\mathbf{u}})\right)\right)-J_{i}\left(\pi_{i}^{n}\left(\sigma_{i}^{n}\left(\bar{u}_{i}\right)\right), \overline{\mathbf{u}}_{-i}\right) \\
& +J_{i}\left(\pi_{i}^{n}\left(\sigma_{i}^{n}\left(\bar{u}_{i}\right)\right), \overline{\mathbf{u}}_{-i}\right)-J_{i}(\overline{\mathbf{u}}) \leq \epsilon_{4}+\epsilon_{5} .
\end{aligned}
$$

This completes the proof since we can choose $\epsilon=\max \left\{\epsilon_{1}, \epsilon_{2}, \epsilon_{4}+\epsilon_{5}\right\}$ arbitrarily small.

Remark 3.5. In fact, the different results established under the umbrella of Theorem 3.1 do not use exactly the same subsets of the Assumptions (A1) to (A6). Indeed, Result (1) requires only Assumptions (A1), (A2) and (A5); Result (2) requires only Assumptions (A2) and (A5); Result (3) requires only Assumptions (A3) and (A4); and Result (4) requires Assumptions (A1) to (A6).

Remark 3.6. In the special case of zero-sum games, the convergence condition (A1) is slightly stronger than the one proposed in Tidball and Altman (1996), and Tidball et al. (1997), ${ }^{\mathrm{a}}$ in which the continuity assumption of (A1a) is replaced by lower-semicontinuity.

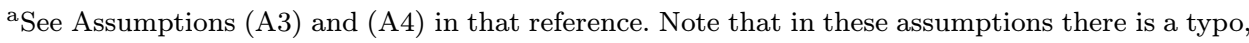
and $u$ and $v$ should be interchanged.
} 


\section{Approximation of a Continuous $m$-Player Game by a Sequence of $m$-Matrix Games}

Let us return to a class of games similar to the Cournot game explored in Sec. 2, and show that we can easily define a good approximating sequence of matrix games.

Consider an $m$-player game $G=(\mathbf{J}, \mathbf{U}), \mathbf{J}=\left(J_{1}, \ldots, J_{m}\right), \mathbf{U}=U_{1} \times U_{2} \cdots U_{m}$, each strategy set $U_{i}$ being endowed with a metric $d_{i}$. Define a sequence $\left(U_{i}^{n}\right)$ of finite subsets of $U_{i}$. For each $n$, define payoff functions $J_{i}^{n}$ as the restriction of the functions $J_{i}$ to the strategy set $\mathbf{U}^{n}=U_{1}^{n} \times \cdots \times U_{i}^{n}$. This defines for each $n$ an $m$-matrix game $G^{n}=\left(\mathbf{J}^{n}, \mathbf{U}^{n}\right)$. Suppose the following is satisfied.

Assumption 4.1. For any $\epsilon>0$ and $u_{i} \in U_{i}$, there exists $N$ such that $\forall n \geq N$, $d\left(u_{i}, U_{i}^{n}\right) \leq \epsilon$.

Then the following holds true.

Proposition 4.1. Suppose that for each $i \in \mathcal{M}, J_{i}$ is continuous. Then the sequence of m-matrix games $G^{n}$ defined above is a good approximating sequence for the game $G$.

Proof. Define the functions $\pi_{i}^{n}$ and $\sigma_{i}^{n}$ as follows

$$
\begin{aligned}
\pi_{i}^{n}: & U_{i}^{n} \rightarrow U_{i} \\
& u_{i} \rightarrow u_{i} \\
\sigma_{i}^{n}: & U_{i} \rightarrow U_{i}^{n} \\
& v_{i} \rightarrow u_{i}^{n} \in \arg \min _{u \in U_{i}^{n}} d_{i}\left(u, v_{i}\right) .
\end{aligned}
$$

From the definition of the functions $\sigma_{i}^{n}$, and by Assumption 4.1, it should be clear that, for any $u_{i} \in U_{i}$, the sequence $u_{i}^{n}=\sigma_{i}^{n}\left(u_{i}\right)$ converges to $u_{i}$. According to the continuity of the functions $J_{i}$ we have

$$
\begin{aligned}
& \lim _{n \rightarrow+\infty}\left[J_{i}\left(\sigma_{i}^{n}\left(u_{i}\right), \mathbf{u}_{-i}\right)-J_{i}\left(u_{i}, \mathbf{u}_{-i}\right)\right]=0, \\
& \lim _{n \rightarrow+\infty}\left[J_{i}\left(\sigma^{n}(\mathbf{u})\right)-J_{i}(\mathbf{u})\right]=0, \\
& \lim _{n \rightarrow+\infty}\left[J_{i}\left(u_{i}, \sigma_{-i}^{n}\left(\mathbf{u}_{-i}\right)\right)-J_{i}\left(u_{i}, \mathbf{u}_{-i}\right)\right]=0,
\end{aligned}
$$

which imply Conditions (A2) to (A6) that define a good approximating sequence. Uniformity of convergence is implied by the compactness of all strategy sets.

\section{Approximation of Games with $S$-Adapted Information Structure}

In this section, we apply the theory of approximation to a class of stochastic dynamic games, played under the $S$-adapted information structure (see Gürkan et al. (1999), Haurie et al. (1987), and Haurie et al. (1990) for an introduction to this type of information structure). 


\subsection{Two-stage games}

We first consider a two-stage $m$-player game. Let $\left(\Omega, 2^{\Omega}, p(\cdot)\right)$ be a finite probability space. At first stage the players have to choose an action, let us denote $\mathbf{a}^{1}=$ $\left(a_{1}^{1}, a_{2}^{1}, \ldots, a_{m}^{1}\right)$ this action profile, where $a_{i}^{1}$ is the action of player $i$, to be chosen in a set $A_{i}^{1}$. Then at a second stage, the players observe the sample value $\omega \in \Omega$ selected according to the probability law $p(\cdot)$. Based on the observation of $\omega$, the players choose a second action. Let us denote $\mathbf{a}^{2}=\left(a_{1}^{2}, a_{2}^{2}, \ldots, a_{m}^{2}\right)$ this action profile, where $a_{i}^{2} \in A_{i}^{2}(\omega)$ is the $i$ th player action at this stage. The sets $A_{i}^{1}$ and $A_{i}^{2}(\omega)$ are assumed to be compact subsets of a normed space.

For given action profiles $\mathbf{a}^{1}, \mathbf{a}^{2}$, and for a sample value $\omega \in \Omega$, the reward received by player $i$ is given by

$$
J_{i}\left(\mathbf{a}^{1}, \mathbf{a}^{2}, \omega\right)=f_{i}^{1}\left(\mathbf{a}^{1}\right)+f_{i}^{2}\left(\mathbf{a}^{1}, \mathbf{a}^{2}, \omega\right)
$$

where $f_{i}^{1}, f_{i}^{2}$ are two real functions.

We call strategies with recourse the class of strategies that corresponds to this information structure, also called $S$-adapted to emphasise the fact that the decisions of players are adapted to the sample realisation of the random element.

For player $i$, such a strategy is defined by the pair $u_{i}=\left(a_{i}^{1}, \alpha_{i}\right)$, where $a_{i}^{1} \in A_{i}^{1}$ and $\alpha_{i}: \omega \mapsto A_{i}^{2}(\omega)$. As usual we denote $\alpha$ the recourse function profile $\left(\alpha_{1}, \alpha_{2}, \ldots, \alpha_{m}\right)$.

For any strategy profile $\mathbf{u}$ we define the payoff function $\mathbf{J}_{i}$ of player $i$ by

$$
\mathbf{J}_{i}(\mathbf{u})=f_{i}^{1}\left(\mathbf{a}^{1}\right)+\mathbb{E}_{p} f_{i}^{2}\left(\mathbf{a}^{1}, \alpha(\omega), \omega\right) .
$$

The approximating sequence $G^{n}$ is obtained by replacing, for each $n$, the sample set $\Omega$ with a subset $\Omega^{n} \subset \Omega$, and by introducing a probability law $p^{n}(\cdot)$ on $\Omega^{n}$. A strategy in $G^{n}$ for player $i$ is thus a pair $u_{1}^{n}=\left(a_{i}^{1}, \alpha_{i}^{n}(\cdot)\right)$, where $a_{i}^{1} \in A_{i}^{1}$ and $\alpha_{i}^{n}(\cdot): \omega^{n} \mapsto A_{i}^{2}\left(\omega^{n}\right)$. A strategy profile $\mathbf{u}^{n}$ is defined as usual. The strategy sets $\mathcal{S}_{i}$ and $\mathcal{S}_{i}^{n}$ for $G$ and $G^{n}$ respectively are endowed with the natural topology. For example, if $u_{i}=\left(a_{i}, \alpha_{i}\right)$ and $u_{i}^{\prime}=\left(a_{i}^{\prime}, \alpha_{i}^{\prime}\right)$ in $\mathcal{S}^{n}$, we use the distance

$$
d^{n}\left(u_{i}, u_{i}^{\prime}\right)=\sup _{\omega \in \Omega^{n}}\left\|\left(a_{i}, \alpha_{i}(\omega)\right)-\left(a_{i}^{\prime}, \alpha_{i}^{\prime}(\omega)\right)\right\| .
$$

We use the following:

\section{Assumption 5.1.}

(C1) The functions $f_{i}^{1}$ and $f_{i}^{2}, i \in \mathcal{M}$ are continuous;

(C2) The functions $f_{i}^{2}$ are bounded by a constant $M$, i.e. $\forall \mathbf{a}^{1}, \mathbf{a}^{2}, \omega f_{i}^{2}\left(\mathbf{a}^{1}, \mathbf{a}^{2}, \omega\right) \leq$ $M$;

(C3) The probabilities on $\Omega$ satisfy $\lim _{n \rightarrow \infty} \sum_{\omega \notin \Omega^{n}} p(\omega)=0$;

(C4) The probabilities on $\Omega$ and $\Omega^{n}$ satisfy $\lim _{n \rightarrow+\infty} \sup _{\omega \in \Omega^{n}}\left|p^{n}(\omega)-p(\omega)\right|=0$.

Remark 5.1. Condition (C1) in Assumption 5.1 implies that Condition (A1) holds for the payoff functions $\mathbf{J}_{i}$ of the game $G$. We also notice that, since $\Omega$ is finite, it only makes sense to consider a sequence where $\Omega^{n} \equiv \Omega$ when $n$ is large 
enough. Then Condition (C3) is trivially satisfied. We nevertheless keep this contourned formulation to prepare for a possible future extension of this type of results to the case of an infinite (countable) probability space. Such an extension is nontrivial since it requires us to drop the assumption of closedness of the strategy sets.

Proposition 5.1. Suppose Assumption 5.1 holds. Then the games $G^{n}$ constitute a good approximating sequence in the sense of Definition 3.3.

Proof. We define the functions $\pi_{i}^{n}, \sigma_{i}^{n}$ as follows

$$
\begin{aligned}
& \pi_{i}^{n}: \mathcal{S}_{i}^{n} \quad \rightarrow \quad \mathcal{S}_{i} \\
& u_{i}=\left(a_{i}, \alpha_{i}\right) \rightarrow \pi_{i}^{n}\left(u_{i}\right)=\left(a_{i}, \tilde{\pi}_{i}^{n}\left(\alpha_{i}\right)\right),
\end{aligned}
$$

where

$$
\begin{aligned}
& \tilde{\pi}_{i}^{n}\left(\alpha_{i}\right)(\omega)=\alpha_{i}(\omega), \quad \text { if } \omega \in \Omega^{n} \\
& \tilde{\pi}_{i}^{n}\left(\alpha_{i}\right)(\omega)=\alpha_{i}\left(\omega^{n}\right), \quad \text { for some fixed } \omega^{n} \in \Omega^{n} \quad \text { if } \omega \notin \Omega^{n},
\end{aligned}
$$

and

$$
\begin{array}{rll}
\sigma_{i}^{n}: \quad \mathcal{S}_{i} & \rightarrow & \mathcal{S}_{i}^{n} \\
u_{i}=\left(b_{i}, \beta_{i}\right) & \rightarrow & \sigma_{i}^{n}\left(u_{i}\right)=\left(b_{i}, \tilde{\sigma}_{i}^{n}\left(\beta_{i}\right)\right),
\end{array}
$$

where $\tilde{\sigma}_{i}^{n}\left(\beta_{i}\right)$ is defined as the restriction of the function $\beta_{i}$ on the set of state $\Omega^{n}$.

Let us denote $v_{i}=\left(b_{i}^{1}, \beta_{i}\right)$ a strategy of player $i$ in $\mathcal{S}_{i}$, and $\mathbf{u}=\left(u_{1}, \ldots, u_{m}\right)$ a strategy profile with $u_{i}=\left(a_{i}^{1}, \alpha_{i}\right) \in \mathcal{S}_{i}^{n}$. Condition (A2) is satisfied since

$$
\begin{aligned}
J_{i}^{n}\left(\sigma_{i}^{n}\left(v_{i}\right), \mathbf{u}_{-i}\right)-J_{i}\left(v_{i}, \pi_{-i}^{n}\left(\mathbf{u}_{-i}\right)\right) \\
=f_{i}^{1}\left(b_{i}^{1}, \mathbf{a}_{-i}^{1}\right)+\mathbb{E}_{p^{n}}\left\{f_{i}^{2}\left(\left(b_{i}^{1}, \mathbf{a}_{-i}^{1}\right),\left(\tilde{\sigma}_{i}^{n}\left(\beta_{i}\right)(\omega), \alpha_{-i}(\omega)\right), \omega\right) \mid \omega \in \Omega^{n}\right\} \\
\quad-f_{i}^{1}\left(b_{i}^{1}, \mathbf{a}_{-i}^{1}\right)-\mathbb{E}_{p}\left\{f_{i}^{2}\left(\left(b_{i}^{1}, \mathbf{a}_{-i}^{1}\right),\left(\beta_{i}(\omega), \tilde{\pi}_{-i}^{n}\left(\alpha_{-i}\right)(\omega)\right), \omega\right) \mid \omega \in \Omega\right\} \\
=\sum_{\omega \in \Omega^{n}}\left(p^{n}(\omega)-p(\omega)\right) f_{i}^{2}\left(\left(b_{i}^{1}, \mathbf{a}_{-i}^{1}\right),\left(\beta_{i}(\omega), \tilde{\alpha}_{-i}(\omega)\right), \omega\right) \\
\quad-\mathbb{E}_{p}\left\{f_{i}^{2}\left(\left(b_{i}^{1}, \mathbf{a}_{-i}^{1}\right),\left(\beta_{i}(\omega), \pi_{-i}^{n}\left(\alpha_{-i}\right)\left(\omega^{n}\right)\right), \omega\right) \mid \omega \in \Omega \backslash \Omega^{n}\right\},
\end{aligned}
$$

where $\omega^{n}$ is some element of $\Omega^{n}$. Thus, according to Conditions (C3) and (C4) and since the reward function $f_{i}^{2}$, at stage 2 is bounded, we have

$$
\lim _{n \rightarrow \infty}\left[J_{i}^{n}\left(\sigma_{i}^{n}\left(v_{i}\right), \mathbf{u}_{-i}\right)-J_{i}\left(v_{i}, \pi_{-i}^{n}\left(\mathbf{u}_{-i}\right)\right)\right]=0
$$

which establishes that Condition (A2) holds. We can show in the same way that Conditions (A3) to (A6) are also satisfied. Indeed for $\mathbf{v}$ such that $v_{i}=\left(b_{i}^{1}, \beta_{i}\right) \in \mathcal{S}_{i}$, and for $\mathbf{u}=\left(u_{1}, \ldots, u_{m}\right)$ such that $u_{i}=\left(a_{i}^{1}, \alpha_{i}\right) \in \mathcal{S}_{i}^{n}$,

$$
\begin{aligned}
J_{i}^{n}\left(\sigma^{n}(\mathbf{v})\right)-J_{i}(\mathbf{v})= & \sum_{\omega \in \Omega^{n}}\left(p^{n}(\omega)-p(\omega)\right) f_{i}^{2}(\mathbf{b}, \beta(\omega), \omega) \\
& -\mathbb{E}_{p}\left\{f_{i}^{2}(\mathbf{b}, \beta(\omega), \omega) \mid \omega \in \Omega \backslash \Omega^{n}\right\}
\end{aligned}
$$




$$
\begin{aligned}
J_{i}^{n}\left(u_{i}, \sigma_{-i}^{n}\left(\mathbf{v}_{-i}\right)\right)-J_{i}\left(\pi_{i}^{n}\left(u_{i}\right), \mathbf{v}_{-i}\right) & \\
= & \sum_{\omega \in \Omega^{n}}\left(p^{n}(\omega)-p(\omega)\right) f_{i}^{2}\left(\left(a_{i}^{1}, \mathbf{b}_{-i}^{1}\right),\left(\alpha_{i}(\omega), \beta_{-i}(\omega)\right), \omega\right) \\
& \quad-\mathbb{E}_{p}\left\{f_{i}^{2}\left(\left(a_{i}^{1}, \mathbf{b}_{-i}^{1}\right),\left(\alpha_{i}\left(\omega^{n}\right), \beta_{-i}(\omega)\right), \omega\right) \mid \omega \in \Omega \backslash \Omega^{n}\right\},
\end{aligned}
$$

and

$$
\begin{aligned}
J_{i}^{n}(\mathbf{u})-J_{i}\left(\pi^{n}(\mathbf{u})\right)= & \sum_{\omega \in \Omega^{n}}\left(p^{n}(\omega)-p(\omega)\right) f_{i}^{2}(\mathbf{a}, \alpha(\omega), \omega) \\
& -\mathbb{E}_{p}\left\{f_{i}^{2}\left(\mathbf{a}, \alpha\left(\omega^{n}\right), \omega\right) \mid \omega \in \Omega \backslash \Omega^{n}\right\} .
\end{aligned}
$$

We get (A3) to (A6) as a consequence of $f_{i}^{2}$ being bounded and Conditions (C3) and $(\mathrm{C} 4)$.

\subsection{K-stage games}

We consider now a game with $K$ stages. At each stage, the players observe the realisation of a discrete stochastic process and choose their respective actions according to the history of the stochastic process.

The dynamics of the stochastic process is defined by the transition matrices $P^{k}, k=1, \ldots, K-1$, where the $(n, m)$ element $P_{n, m}^{k}$ is the probability for the stochastic state variable to jump from state $n$ at stage $k$ to state $m$ at stage $k+1$. So to any realisation of the stochastic sequence, from stage 1 to stage $K-1$, $\omega=\left(\omega^{1}, \omega^{2} \cdots \omega^{K-1}\right)$, is associated a probability

$$
p(\omega)=p^{1}\left(\omega^{1}\right) \prod_{k=1}^{K-2} P_{\omega^{k} \omega^{k+1}}^{k},
$$

where $p^{1}\left(\omega^{1}\right)$ is probability distribution at stage 1 .

Definition 5.1. A sequence $\omega=\left(\omega^{1}, \omega^{2} \cdots \omega^{K-1}\right)$ is also called a scenario. A scenario is feasible if $p(\omega)$ [defined by (23)] is strictly positive. We denote $W$ the set of feasible scenarios.

A strategy $u_{i}$ of player $i$ will be a vector $\alpha_{i}^{1}, \alpha_{i}^{2}, \ldots, \alpha_{i}^{K}$, where $\alpha_{i}^{k}: \omega \mapsto A_{i}^{k}(\omega)$ is a function that associates with any realisation of the stochastic variable at stage $k$ the action chosen by the player $i$ at stage $k$ in the compact set $A_{i}^{k}(\omega) \subset A_{i}^{k}$. These strategy sets can be endowed with the uniform convergence (in $\omega$ ) topology.

We denote $\mathcal{S}_{i}$ a set of admissible mappings from the set of feasible scenarios $W$ to the set $\mathcal{A}_{i}^{K-1}$ of action sequences of player $i$. Any element $u_{i}$ of $\mathcal{S}_{i}$ must satisfy the following non-anticipativeness condition:

Assumption 5.2. For any two feasible scenarios $\omega=\left(\omega^{1}, \omega^{2} \cdots \omega^{K-1}\right)$ and $\bar{\omega}=$ $\left(\bar{\omega}^{1}, \bar{\omega}^{2} \cdots \bar{\omega}^{K-1}\right)$, if $\omega^{k}=\bar{\omega}^{k}$, for $k$ from 1 to $\bar{k}$, then $a_{i}^{\bar{k}}=\left(u_{i}(\omega)\right)^{\bar{k}}=\left(u_{i}(\bar{\omega})\right)^{\bar{k}}$. 
This assumption implies that the decisions at stage $\bar{k}$ are adapted to the realisation $\omega^{1}, \ldots, \omega^{\bar{k}}$ of the stochastic-state variable. However, the decision of a player is not adapted to the realisation of the decision process of the other players. This is consistent with the $S$-adapted information structure, introduced in Haurie et al. (1990) and further studied in Haurie and Moresino (to appear).

Since the choice of actions of player $i$ is not affected by the choice of any of his opponent, there exists a one to one mapping from the set $\mathcal{S}_{i}$ of strategies with recourse to the set $\overline{\mathcal{S}}_{i}$. This mapping associates with the strategy $u_{i}=\left(\alpha_{i}^{1}, \alpha_{i}^{2} \cdots \alpha_{i}^{K-1}\right)$ of $\mathcal{S}_{i}$, the non-anticipative function, $\bar{u}_{i}$ defined by

$$
\begin{aligned}
\bar{u}_{i}: \Omega & \rightarrow \mathcal{A}_{i}^{K-1}, \quad \text { such that } \bar{u}_{i}\left(\omega^{1}, \omega^{2} \cdots \omega^{K-1}\right) \\
& =\left(a_{i}^{1}, a_{i}^{2} \cdots a_{i}^{K-1}\right), \quad \text { with } a_{i}^{k}=\alpha_{i}^{k}\left(\omega^{k}\right) .
\end{aligned}
$$

For any choice of a strategy profile $\mathbf{u}=\left(u_{1}, u_{2} \cdots u_{m}\right)$, and any feasible scenario $\omega$, the player $i$ receives the payment

$$
J_{i}(\mathbf{u}, \omega)=\sum_{k=1}^{K-1} f_{i}^{k}\left(\mathbf{x}^{k}, \alpha_{i}^{k}\left(\omega^{k}\right), \omega^{k}\right),
$$

where the player $i$ 's state variable is determined by the evolution equation

$$
x_{i}^{k+1}=g_{i}\left(x_{i}^{k}, \alpha_{i}^{k}\left(\omega^{k}\right)\right),
$$

where $g_{i}(\cdot)$ are continuous functions with bounded values. Equivalently, if $\mathbf{u}=$ $\left(\bar{u}_{1}, \bar{u}_{2} \cdots \bar{u}_{m}\right)$ is the corresponding non-anticipative function profile, the player $i$ 's payment is defined as

$$
J_{i}(\mathbf{u}, \omega)=\sum_{k=1}^{K-1} f_{i}^{k}\left(\mathbf{x}^{k},\left(u_{i}(\omega)\right)^{k}, \omega^{k}\right),
$$

where

$$
x_{i}^{k+1}=g_{i}\left(x_{i}^{k},\left(u_{i}(\omega)\right)^{k}\right) .
$$

Again, for a strategy profile $\mathbf{u}$ the evaluation function is defined as the expected value of $J_{i}(\mathbf{u}, \omega)$,

$$
\mathbf{J}_{i}(\mathbf{u})=\mathbb{E}_{p} J_{i}(\mathbf{u}, \omega) .
$$

With the use of non-anticipative functions as strategies, the game can thus be formulated in its normal form.

We define an approximating sequence of games $G^{n}$, where $G^{n}$ is played with an event tree of feasible scenarios $W^{n}$ defined as a subset $W$. The probability of a scenario in $W^{n}$ is given by a probability law $p^{n}(\cdot)$. Let us denote $\overline{\mathcal{S}}_{i}^{n}$ the set of strategies of player $i$ in the game $G^{n}$. 
We use the following:

\section{Assumption 5.3.}

(D1) The functions $f_{i}^{k}, i \in \mathcal{M}, k=1,2, \ldots, K-1$ are continuous;

(D2) The functions $f_{i}^{k}, i \in \mathcal{M}, k=1,2, \ldots, K-1$ are bounded by a constant $M$;

(D3) The probabilities on $W$ satisfy $\lim _{n \rightarrow \infty} \sum_{\omega \in W \backslash W^{n}} p(\omega)=0$;

(D4) The probabilities on $W$ and $W^{n}$ satisfy $\lim _{n \rightarrow \infty} \sup _{\omega \in W^{n}}\left|p^{n}(\omega)-p(\omega)\right|=0$.

To establish:

Proposition 5.2. Assume that Assumption 5.3 holds. Then $\left\{G^{n}\right\} n \in \mathbb{N}$ is a good approximating sequence in the sense of Definition 3.3, and Theorem 3.1 applies.

Remark 5.2. Condition (D1) in Assumption 5.3 implies that Condition (A1) is satisfied for the payoff functions $\mathbf{J}_{i}$ of the game $G$. Notice also that, if $W$ is a finite set, Condition (D3) implies that $W_{n} \equiv W$ if $n$ is large enough.

Proof. We define the functions $\pi_{i}^{n}$ and $\sigma_{i}^{n}$ as follows:

$$
\begin{aligned}
\pi_{i}^{n}: & \mathcal{S}_{i}^{n} \rightarrow \mathcal{S}_{i} \\
& u_{i} \rightarrow \pi_{i}^{n}\left(u_{i}\right)
\end{aligned}
$$

where

$$
\begin{aligned}
& \pi_{i}^{n}\left(u_{i}\right)(\omega)=u_{i}(\omega), \quad \text { if } \omega \in W^{n} \\
& \pi_{i}^{n}\left(u_{i}\right)(\omega)=u_{i}\left(\omega^{n}\right), \quad \text { if } \omega \notin W^{n} .
\end{aligned}
$$

Here, $\omega^{n}$ is any given scenario in $W^{n}$,

$$
\begin{aligned}
\sigma_{i}^{n}: & \mathcal{S}_{i} \rightarrow \mathcal{S}_{i}^{n} \\
v_{i} & \rightarrow \sigma_{i}^{n}\left(v_{i}\right),
\end{aligned}
$$

where $\sigma_{i}^{n}\left(v_{i}\right)$ is defined as the restriction of the function $v_{i}$ on the set $W^{n}$ of feasible scenarios of game $G^{n}$.

The proof that (A2) to (A6) holds is similar as in Proposition 5.1. Uniformity in convergence is obtained due to compactness of all strategy sets.

\subsection{A numerical illustration}

We consider a duopoly game where two firms supply a market for a homogeneous good. The state variable $x_{i}^{k+1}$ describes the production capacity of firm $i$ at stage $k$, and is determined by the evolution equation

$$
x_{i}^{k+1}=g_{i}\left(x_{i}^{k},\left(\bar{u}_{i}(\omega)\right)^{k}\right)=\left(\bar{u}_{i}(\omega)\right)^{k}+\left(1-\beta_{i}\right) x_{i}^{k},
$$


where $\left(\bar{u}_{i}(\omega)\right)^{k}$ represents the investment in production capacity for firm $i$ at stage $k$, whereas $\beta_{i}$ is the capacity depreciation rate for firm $i$. For the numerical illustration, the depreciation rates are $\beta_{1}=0.08$ and $\beta_{2}=0.06$. The admissible controls are those which keep the capacity non-negative.

The stochastic state of the market is represented by a discrete-state Markov chain. For the numerical illustration, we assume that the market can be in one of three possible states, $\Omega=\{1,2,3\}$. We assume that the inverse demand law at stage $k$ depends on the market condition $\omega^{k}$ in the following way

$$
D\left(x_{1}^{k}+x_{2}^{k}, \omega^{k}\right)=\frac{a\left(\omega^{k}\right)}{x_{1}^{k}+x_{2}^{k}+b\left(\omega^{k}\right)}-c\left(\omega^{k}\right) .
$$

Here $D$ is the market clearing price, given the total supply $x_{1}^{k}+x_{2}^{k}$. The coefficients are: $a(1)=120, a(2)=100, a(3)=80, b(1)=b(2)=b(3)=20, c(1)=3$, $c(2)=2.5$ and $c(3)=2$. The dynamics of the Markov chain is described by the following transition matrix:

$$
P^{k}=\left(\begin{array}{ccc}
e^{-0.2} & 1-e^{-0.2} & 0 \\
\left(1-e^{-0.05}\right) \frac{0.01}{0.05} & e^{-0.05} & \left(1-e^{-0.05}\right) \frac{0.04}{0.05} \\
1-e^{-0.1} & 0 & e^{-0.1}
\end{array}\right)
$$

for all $k=1, \ldots, K-1$. We also assume that each firm has a quadratic maintenance and investment cost. So the profit functions at stage $k$ are given by

$$
f_{i}^{k}\left(\mathbf{x}^{k},\left(\bar{u}_{i}(\omega)\right)^{k}, \omega^{k}\right)=e^{-\rho_{i} k}\left[D\left(x_{1}^{k}+x_{2}^{k}, \omega^{k}\right) x_{i}^{k}-\left(x_{i}^{k}\right)^{2}-\left(\left(\bar{u}_{i}(\omega)\right)^{k}\right)^{2}\right], \quad i=1,2 .
$$

The discount rates are $\rho_{1}=\rho_{2}=0.09$ and the time horizon is $K=10$.

This is the discrete time duopoly studied in Haurie and Moresino (to appear). Theorem 7 therein, establishes existence and uniqueness for the Nash equilibrium. This dynamic game exhibits a so-called "turnpike property" which means that, for each discrete state, an attractor called "turnpike", exists for the optimal trajectory of the production capacity of each firm.

The game is defined over a finite event tree, hence we are in the case where $\Omega$ is finite. The approximating sequence of probability measures that we use is obtained through a statistical sampling procedure. The value $p^{n}(\omega)$ is the sampling frequency of scenario $\omega$ for a sample size $n$. Note that, in this framework, the probability measure for the first approximating game $G^{1}$ is given by a single scenario $\omega$ having a probability 1 . As $n$ increases, the set of scenarios with nonzero probabilities will become larger since the approximating relative frequencies converge almost surely to the scenario probabilities of $G$. With probability 1, after a finite number of trials, the set $W^{n}$ of feasible scenarios in $G^{n}$ will be equal to $W$. By the strong law of large numbers, Assumptions (D3) and (D4) are satisfied here, in the sense of almost sure convergence. With probability 1 , the sequence $G^{n}$ will be a good approximating sequence. 
To illustrate the convergence of the presented sampling method, we compute the turnpike values when the market is in state 1 , for two different sample size, namely $n=100$ and $n=10000$. In each case, ten different samples have been randomly drawn and the $S$-adapted equilibra computed for the sampled event trees.

For the game $G$ the turnpike values, computed through a direct method, are 0.927 for firm 1 and 0.931 for firm 2 . The results for the approximating games are summarised in Tables 3 and 4 which clearly show convergence.

Table 3. Turnpikes for the approximating game with sample size $n=100$.

\begin{tabular}{lcc}
\hline \hline & Turnpike firm 1 & Turnpike firm 2 \\
\hline Sample C1 & 0.927 & 0.931 \\
Sample C2 & 0.925 & 0.928 \\
Sample C3 & 0.927 & 0.931 \\
Sample C4 & 0.927 & 0.931 \\
Sample C5 & 0.927 & 0.930 \\
Sample C6 & 0.926 & 0.930 \\
Sample C7 & 0.927 & 0.930 \\
Sample C8 & 0.928 & 0.932 \\
Sample C9 & 0.926 & 0.930 \\
Sample C10 & 0.927 & 0.930 \\
Mean & 0.927 & 0.930 \\
Standard deviation & 0.0078 & 0.0100 \\
\hline \hline
\end{tabular}

Table 4. Turnpikes for the approximating game with sample size $n=10000$.

\begin{tabular}{lcc}
\hline \hline & Turnpike firm 1 & Turnpike firm 2 \\
\hline Sample M1 & 0.927 & 0.931 \\
Sample M2 & 0.928 & 0.931 \\
Sample M3 & 0.927 & 0.930 \\
Sample M4 & 0.927 & 0.930 \\
Sample M5 & 0.927 & 0.931 \\
Sample M6 & 0.928 & 0.931 \\
Sample M7 & 0.927 & 0.931 \\
Sample M8 & 0.928 & 0.931 \\
Sample M9 & 0.927 & 0.931 \\
Sample M10 & 0.928 & 0.931 \\
Mean & 0.927 & 0.931 \\
Standard deviation & 0.00049 & 0.00040 \\
\hline \hline
\end{tabular}




\section{Conclusion}

In this paper, we have provided conditions which imply that a sequence of approximating games will have $(\epsilon)$ equilibria that approximate an $\left(\epsilon^{\prime}\right)$ equilibrium in the limit game. These results have been illustrated on a static Cournot duopoly game and on a stochastic version of the Cournot game with the $S$-adapted information structure.

\section{Acknowledgment}

This research has been supported by The Swiss Science Foundation (FNRS).

\section{References}

Audet, C., P. Hansen, B. Jaumard and G. Savard (1999). A Symmetrical Linear Maximin Approach to Disjoint Bilinear Programming, Mathematical Programming, Vol. 85, No. 3, 573-592.

Cavazzuti, E. and N. Pacchiarotti (1986). Convergence for Nash Equilibria. Bollettino U.M.I., 5-B, 247-266.

Gürkan, G., Y. Özge and S. M. Robinson (1999). "Sample Path Solution of Stochastic Variational Inequalities". Mathematical Programming, Vol. 84, 313-333.

Haurie, A. and F. Moresino. "Computation of $S$-Adapted Equilibria in Piecewise Deterministic Games Via Stochastic Programming Methods". Annals of the International Society of Dynamic Games, Vol. 6 (to appear).

Haurie, A., G. Zaccour, J. Legrand and Y. Smeers (1987). "A Stochastic Dynamic NashCournot Model for The European Gas Market". Tech. Report HEC Montreal.

Haurie, A., G. Zaccour and Y. Smeers (1990). "Stochastic Equilibrium Programming for Dynamic Oligopolistic Markets". Journal of Optimization Theory and Applications, Vol. 66, 243-253.

Morgan, J. and R. Raucci (1999). "New Convergence Results for Nash Equilibria". Journal of Convex Analysis, Vol. 6, No. 2, 377-385.

Rosen, J. B. (1965). "Existence and Uniqueness of the Equilibrium Points for Concave $N$-Person Games". Econometrica, Vol. 33. No. 3.

Tidball, M. and E. Altman, "Approximations in Dynamic Zero-Sum Games I". SIAM Journal on Control and Optimization, Vol. 34, 311-328.

Tidball, M., O. Pourtallier and E. Altman (1997). "Approximations in Dynamic Zero-sum Games II". SIAM Journal on Control and Optimization, Vol. 35, No. 6, 2101-2117.

Whitt, W. (1980). "Finite State Approximations for Denumerable State Infinite Horizon Discounted Markov Decision Processes". Journal of Mathematical Analysis and Applications, Vol. 74, 292-295; "Representation and Approximation of NonCooperative Sequential Games". SIAM Journal on Control and Optimization, Vol. 18, 33-43. 